\title{
Calcul d'écoulements incompressibles derrière des obstacles et analyse des solutions transitoires
}

\author{
Charles-Henri Bruneau*
}

\begin{abstract}
Résumé
Le but de ce papier est de mettre l'accent sur les difficultés majeures que l'on rencontre pour simuler des écoulements transitoires de fluides incompressibles derrière des obstacles ainsi que pour analyser les solutions obtenues. Il est hors de question de prétendre à l'exhaustivité, seuls certains aspects sont présentés ici. En premier lieu les conditions aux limites artificielles du domaine de calcul et la prise en compte des obstacles. Ces deux points conditionnent pour beaucoup le choix de l'approximation selon le maillage utilisé et l'aptitude à calculer des solutions à nombres de Reynolds élevés. C'est à dire des solutions transitoires vers la turbulence. Là réside un nouvel écueil car il est très difficile de qualifier précisément une solution avec des outils d'analyse classique dès qu'elle n'est plus strictement périodique.
\end{abstract}

\section{Introduction}

Il est impossible de faire une revue exhaustive de toutes les méthodes mises en œuvre pour approcher les équations de Navier-Stokes régissant l'écoulement d'un fluide incompressible tellement les chercheurs du monde entier ont été prolixes sur le sujet depuis trente ans. Ils ont fait montre d'une grande imagination et toutes les méthodes inventées ont été appliquées à la résolution de ces équations. On peut d'ailleurs s'en convaincre à la lecture des nombreux livres $[7,12,13,19,28,33,34]$ ou en participant à une des conférences internationales qui traite en tout ou partie du sujet $[18,32,25,10,17]$. On est encore loin de pouvoir calculer un écoulement turbulent par simulation directe des équations de Navier-Stokes et les nombres de Reynolds que l'on peut traiter sont encore loins de la réalité physique. Cependant, il y a quelques progrès de faits en parallèle avec le développement des moyens de calcul qui laissent espérer que cela sera possible dans le futur. Nous présentons ici quelques aspects des recherches récentes effectuées sur les conditions aux limites artificielles du domaine de calcul, la représentation des obstacles, l'approximation numérique, les méthodes de résolution et l'analyse des solutions calculées. Nous ne prétendons nullement que les choix présentés sont toujours optimaux mais ils correspondent à une réalité et permettent de faire des simulations proches des limites de l'état de l'art actuel. Il est en effet possible de calculer des solutions transitoires en 2D et même en 3D si l'on tire parti au mieux des ordinateurs modernes. On est alors confronté à une nouvelle difficulté qui est l'analyse des solutions. Des outils classiques comme la transformée de Fourier qualifient bien les solutions périodiques mais il est toujours aussi difficile d'analyser ce qui se passe au delà. Le processus de transition vers le chaos et la turbulence demeure un peu mystérieux faute d'outils d'analyse suffisamment puissants.

"Mathématiques Appliquées de Bordeaux, Université Bordeaux 1, 351 cours de la Libération, F-33405 Talence, bruneau@math.u-bordeaux.fr 


\section{Le modèle de Navier-Stokes}

\subsection{Les équations}

A partir des équations de conservation de la mécanique des milieux continus, il est facile d'obtenir les équations de Navier-Stokes régissant l'écoulement d'un fluide newtonien incompressible dans un domaine $\Omega \subset \mathbb{R}^{N}$ avec $N \leq 3$

$$
\begin{aligned}
\partial_{t} U+(U \cdot \nabla) U-\frac{1}{R e} \Delta U+\nabla p & =F & & \text { dans } \Omega_{T}=\Omega \times(0, T) \\
\operatorname{div} U & =0 & & \text { dans } \Omega_{T} .
\end{aligned}
$$

La première équation peut s'écrire

$$
\partial_{t} U+(U \cdot \nabla) U-\operatorname{div} \tilde{\sigma}(U, p)=F \quad \operatorname{dans} \Omega_{T}
$$

ou bien

$$
\partial_{t} U+(U \cdot \nabla) U-\operatorname{div} \sigma(U, p)=F \quad \operatorname{dans} \Omega_{T}
$$

où $\tilde{\sigma}(U, p)$ et $\sigma(U, p)$ sont respectivement le pseudo tenseur des contraintes et le tenseur des contraintes définis par:

$$
\begin{gathered}
\tilde{\sigma}(U, p)=\frac{1}{R e} \nabla U-p I \\
\sigma(U, p)=\frac{2}{\operatorname{Re}} D(U)-p I \text { avec } D(U)_{i j}=\frac{1}{2}\left(\frac{\partial u_{i}}{\partial x_{j}}+\frac{\partial u_{j}}{\partial x_{i}}\right)
\end{gathered}
$$

où $U=\left(u_{i}\right)_{i}$ est le vecteur vitesse, $p$ la pression, Re le nombre de Reynolds adimensionné et $F$ représente les forces extérieures. Dans la plupart des cas $F=0$ et le mouvement est induit par une condition aux limites de Dirichlet non homogène imposée sur une partie $\Gamma_{D}$ du bord $\partial \Omega$.

Ces équations en variables primitives vitesse-pression peuvent être transformées en faisant apparaitre le rotationnel $\omega=\nabla \wedge U$. Ce qui donne:

$$
\begin{aligned}
\partial_{t} \omega+(U \cdot \nabla) \omega-\frac{1}{R e} \Delta \omega & =(\omega \cdot \nabla) U+\nabla \wedge F & & \text { dans } \Omega_{T} \\
\nabla \wedge U & =\omega & & \text { dans } \Omega_{T} \\
\operatorname{div} U & =0 & & \text { dans } \Omega_{T}
\end{aligned}
$$

où une partie du terme de convection est traitée comme un terme source dans la première équation; en dimension deux ce terme est nul. Il y a d'autres formulations possibles, en particulier en dimension deux en faisant apparaitre la fonction de courant (voir [15] pour une discussion plus complète).

\subsection{La donnée initiale}

Les équations d'évolution (1) (2) nécessitent l'ajout d'une condition initiale

$$
U(x, 0)=U_{0}(x) \text { dans } \Omega
$$


où d'un point de vue mathématique $U_{0}$ doit appartenir au bon espace, en particulier $U_{0}$ doit a priori satisfaire les conditions aux limites et être à divergence nulle. Ce qui n'est pas si facile à réaliser en pratique. L'expérience numérique montre que ce n'est pas obligatoire et que cela est automatiquement induit par les premiers pas de temps.

Une autre question liée à la condition initiale est l'utilisation de schémas en temps d'ordre élevé qui nécessitent plusieurs données initiales $U^{-j}(x), 0 \leq j \leq J$ pour démarrer le calcul. Une solution très simple est de poser $U^{-j}(x)=U_{0}(x)$ pour $0 \leq j \leq J$. On peut aussi utiliser un schéma d'Euler aux premiers pas de temps mais cela n'améliore pas notablement le calcul.

\subsection{Les conditions aux limites}

Dans tous les problèmes d'équations aux dérivées partielles il est indispensable de bien traiter les conditions aux limites. Ici, elles sont de trois types: d'entrée, d'adhérence sur les parois rigides et de sortie ou ouvertes sur les frontières artificielles du domaine de calcul. Dans les deux premiers cas, il suffit d'imposer des conditions de Dirichlet. Par contre, le dernier cas n'est pas trivial et est à lui seul générateur d'une importante prose et de nombreux tests $[31,4]$. Ces trois types de condition sont indispensables par exemple si on veut calculer l'écoulement confiné ou non derrière un obstacle. Voir la figure 1 où le domaine $\Omega$ a pour bord $\partial \Omega=\Gamma_{D} \cup \Gamma_{0} \cup \Gamma_{1} \cup \Gamma_{N}$.



FIG. 1 - Domaine de calcul

Sur $\Gamma_{D}$ l'écoulement infini amont $\left(U_{\infty}, p_{\infty}\right)$ est imposé. Si l'écoulement est libre il suffit d'imposer un écoulement constant et si l'écoulement est confiné on impose un écoulement de Poiseuille à l'entrée. Sur $\Gamma_{1}$ il ya une condition d'adhérence $U=0$ comme sur $\Gamma_{0}$ si le maillage est adapté à la géométrie de l'obstacle. on verra au paragraphe suivant qu'il y a d'autres possibilités pour représenter l'obstacle. Quant à la condition a imposer sur $\Gamma_{N}$ elle est loin d'être évidente. En effet si $\Gamma_{D}$ n'est pas trop prés de l'obstacle $\Omega_{0}$, la condition de Dirichlet en entrée est correcte car elle ne génère pas de perturbations. Par contre, même si la frontière avale est relativement éloignée de $\Omega_{0}$ la plupart des conditions aux limites produisent de fortes réflexions des tourbillons convectés par l'écoulement sur la limite artificielle du domaine de calcul. Comme cela est dû uniquement à la limitation imposée par les moyens de calcul, il ne faut pas compter sur la physique pour nous dire quelle est la bonne condition qui permet d'obtenir sur le domaine tronqué la restriction de la solution sur le domaine infini. les numériciens utilisent essentiellement deux voies. Soit ils rajoutent derrière le domaine de calcul un zone tampon à l’intérieur de laquelle les équations sont 
modifiées [9, 30], soit ils imposent les meilleures conditions connues. Il y a eu de nombreux travaux dans ce sens et nous renvoyons à [31] et aux nombreuses références bibliographiques que contient cette excellente revue pour une présentation plus complète. La condition la plus utilisée est sans conteste la condition de traction nulle $\sigma(U, p) n=0$ que nous généralisons dans [4] par

$$
\tilde{\sigma}(U, p) n=\tilde{\sigma}\left(U_{\infty}, p_{\infty}\right) n
$$

ou bien

$$
\sigma(U, p) n=\sigma\left(U_{\infty}, p_{\infty}\right) n
$$

pour un écoulement de Stokes et par

$$
\tilde{\sigma}(U, p) n+\frac{1}{2}(U \cdot n)^{-}\left(U-U_{\infty}\right)=\tilde{\sigma}\left(U_{\infty}, p_{\infty}\right) n
$$

ou bien

$$
\sigma(U, p) n+\frac{1}{2}(U \cdot n)^{-}\left(U-U_{\infty}\right)=\sigma\left(U_{\infty}, p_{\infty}\right) n
$$

pour un écoulement de Navier-Stokes avec la notation $a=a^{+}-a^{-}$. Le terme inertiel supplémentaire est nul sauf si U.n est négatif, de façon à faciliter la convection des tourbillons et supprimer les réflexions quand l'écoulement est rentrant. A partir d'une formulation mixte, on peut montrer par la méthode de l'énergie que les conditions (9) ou bien (10) conduisent à un problème bien posé [3]. Ces conditions sont de plus en plus utilisées dans les centres de recherche et l'industrie.

\subsection{L'obstacle}

La méthode la plus classique pour représenter l'obstacle est de construire un maillage qui approche au mieux sa frontière $\Gamma_{0}$. Cela induit le plus souvent l'utilisation d'un maillage non structuré et force le choix de l'approximation mais la condition d'adhérence peut être imposée exactement aux points de discrétisation sur $\Gamma_{0}$. Si l'on veut utiliser des méthodes spectrales ou aux différences finies sur maillage cartésien, il faut trouver un autre moyen de prendre en compte l'obstacle. En d'autres termes il faut forcer la condition d'adhérence à la paroi ou bien l'écoulement a être nul à l'intérieur de l'obstacle. A la suite de [27], divers auteurs ont ajouté deux termes de pénalisation de la vitesse et de son intégrale en temps sur $\Gamma_{0}$ dans l'équation de conservation de la quantité de mouvement. Les points définissant $\Gamma_{0}$ sont soit les sommets les plus proches, soit les points d'intersection de la surface avec les lignes de maillage. Dans ce dernier cas, les interpolations doivent être bien choisies [14, 30]. Il apparait de surcroit qu'à grands nombres de Reynolds il faut pénaliser sur le volume de l'obstacle et non seulement sur sa surface pour obtenir des solutions correctes [30]. Une autre idée est d'assimiler l'obstacle à un milieu poreux de perméabilité très faible $K$. Cela revient d'une certaine façon à résoudre les équations de Navier-Stokes dans le fluide et les équations de Darcy dans le solide. Un moyen d'y parvenir est de pénaliser la vitesse ou l'équation de Darcy dans le solide. C'est à dire de remplacer les équations (1),(2) par

$$
\begin{array}{rll}
\partial_{t} U+(U \cdot \nabla) U-\frac{1}{R e} \Delta U+\frac{U}{K}+\nabla p & =F \quad & \text { dans } D_{T}=D \times(0, T) \\
\operatorname{div} U & =0 \quad \text { dans } D_{T}
\end{array}
$$

ou bien

$$
\begin{array}{rll}
\partial_{t} U+(U \cdot \nabla) U-\frac{1}{R e} \Delta U+\frac{1}{K}\left(\frac{U}{K}+\nabla p\right)+\nabla p & =F & \text { dans } D_{T}=D \times(0, T) \\
\operatorname{div} U & =0 & \text { dans } D_{T}
\end{array}
$$

ESAIM: Proc., VoL. 3, 1998, 37-50 
sur $D=\Omega \cup \Gamma_{0} \cup \Omega_{0}$ où $K$ est pris très grand dans le fluide et petit dans le solide. On peut montrer alors que la solution de (11),(12) converge vers la solution des équations de NavierStokes et des tests numériques montrent que pour $K=10^{-8}$ dans le solide les solutions sont indiscernables [1]. Evidemment, cela rajoute des calculs puisqu'il faut résoudre les équations (11), (12) en plus dans $\Omega_{0}$. Cependant ce surcoût est en général négligeable devant le gain réalisé par l'utilisation d'un maillage cartésien. De plus, le champ de pression est calculé dans $\Omega_{0}$, ce qui permet de déterminer les forces de traînée et de portance [6].

\section{Simulation numérique}

\subsection{L'approximation}

De nombreux auteurs ont écrit des livres entiers pour décrire un type d'approximation des équations de Navier-Stokes [7, 12, 13, 19, 28, 33, 34]. Il n'est donc pas dans notre propos d'essayer de résumer ces livres en quelques lignes mais plutôt de mettre l'accent sur les difficultés majeures que sont l'équilibre entre les termes de convection et de diffusion et la contrainte de divergence nulle. Il est maintenant reconnu que les termes de convection doivent être traités explicitement pour éviter l'ajout d'un terme de diffusion numérique en temps. Cela signifie que ces termes sont exprimés au temps $n \delta t$ pour calculer la solution au temps $(n+1) \delta t$. Par contre, tous les autres termes peuvent être discrétisés implicitement au temps $(n+1) \delta t$. L'approximation elle-même ne semble pas être déterminante et il est possible de calculer des solutions correctes quel que soit le choix réalisé. Ce choix est plus lié au maillage et donc à la méthode utilisée pour prendre en compte l'obstacle. Sur maillage non structuré c'est souvent la méthode des volumes finis qui est utilisée [26]. Avec pénalisation de l'obstacle il est possible d'utiliser des différences finies [5] ou une méthode spectrale [21,30]. Dans tous les cas, on obtient un système linéaire assez bien conditionné et facile à inverser car les termes de convection sont reportés au second membre.

Il est impératif d'apporter un soin particulier à la discrétisation des termes de convection. Cela garantit en effet à la fois la stabilité et la précision du calcul. Il est important de remarquer que toute diffusion numérique apportée par le schéma d'approximation vient s'ajouter à $-\frac{1}{R e} \Delta U$ et donc change la valeur du nombre de Reynolds. Par exemple, la discrétisation de

$$
u \frac{\partial u}{\partial x}
$$

au point $j$ en dimension un par un schéma décentré du premier ordre

$$
u_{j}^{n}\left(u_{j}^{n}-u_{j-1}^{n}\right) / \delta x
$$

dans le cas où $u_{j}^{n}$ est positif correspond à une approximation du second ordre de

$$
u \frac{\partial u}{\partial x}-\frac{\delta x}{2} \frac{\partial}{\partial x}\left(|u| \frac{\partial u}{\partial x}\right)
$$

et donc le nombre de Reynolds est diminué. Ce qui a pour effet de stabiliser le processus de calcul mais supprime tout sens quantitatif aux résultats. Seule une analyse qualitative de la solution en fonction de la croissance du nombre de Reynolds reste alors possible. Encore aujourd'hui il est difficile de dire quel est le nombre de Reynolds critique pour lequel il y a perte de stabilité de la solution stationnaire pour le problème de la cavité entrainée. Ceci parce qu'il est voisin de $10^{4}$ et proche des limites du calcul, au moins il n'est pas facile d'obtenir une grande précision pour de telles valeurs. Il est cependant possible d'écrire des schémas moins diffusifs que (15). On peut par exemple le remplacer par

$$
u_{j-1 / 2}^{n}\left(4 u_{j}^{n}-5 u_{j-1}^{n}+u_{j-2}^{n}\right) / 3 \delta x-u_{j+1 / 2}^{n}\left(4 u_{j}^{n}-5 u_{j+1}^{n}+u_{j+2}^{n}\right) / 3 \delta x
$$


si $u_{j-1 / 2}^{n}$ est positif et $u_{j+1 / 2}^{n}$ est negatif. Les résultats numériques présentés au chapitre 4 sont obtenus avec les schéma ci-dessus. Dans le cas de la cavité entrainée, cela permet de situer la première bifurcation de Hopf aux alentours de 7500 ; ce qui semble être une bonne valeur du nombre de Reynolds critique comme en témoigne les travaux récents sur le sujet $[25,17]$. Les autres résultats sont obtenus généralement avec des schémas compacts d'ordre élevé.

L'autre point clé est la prise en compte de la contrainte de divergence nulle et de nombreuses méthodes sont envisageables. En éléments finis, il y a eu diverses constructions de l'espace approché où la contrainte (2) est satisfaite au sens fort ou en un sens faible [33]. En différences finies, un moyen très simple d'approcher (2) est d'utiliser un schéma centré aux points en pression d'une grille décalée (figure 2). Ce qui permet de satisfaire la condition sur une maille sans interpolation et de relier les inconnues.

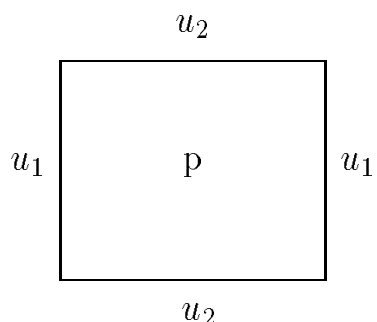

FIG. 2 - Une maille avec ses inconnues décalées

Parmi les autres possibilités, la plus célèbre est sans aucun doute la méthode de dualité évoquée ci-dessous.

\subsection{La convergence}

Le dernier point est le processus de résolution global qui conditionne la vitesse de convergence à chaque pas de temps et donc la faculté de suivre les solutions au cours de simulations en temps longs. Un des choix possibles est de considérer les équations de Navier-Stokes comme un problème de minimisation avec contrainte où la pression joue le rôle de multiplicateur de Lagrange. La convergence est obtenue par l'algorithme d'Uzawa auquel est associé une méthode de gradient préconditionné performante pour l'inversion du système linéaire.

Une autre voie est de décomposer la solution selon ses différentes échelles. Dans la méthode de Galerkin non linéaire ces échelles sont séparées en deux parties, grandes et petites, qui sont capturées simultanément. Dans l'état actuel cela permet d'obtenir un gain de performances significatif par rapport à la méthode classique quelle que soit l'approximation utilisée. Dans le même ordre d'idées, la méthode multigrille est un outil très puissant [2, 16]. En effet, avec une simple méthode itérative de relaxation, il est très facile de capturer les échelles liées à une grille. Donc en utilisant une suite de grilles contenant en particulier des grilles très grossières, on peut capturer rapidement l'ensemble des échelles couvertes par toutes les grilles, y compris les basses fréquences ; la grille la plus fine donnant la limitation des hautes fréquences accessibles [5, 37].

Dans tous les cas, il faut viser la performance maximale car pour faire des simulations directes de la transition vers la turbulence, il faut à la fois utiliser des grilles très fines et calculer la solution sur de longs intervalles de temps. Aujourd'hui une simulation à nombre de Reynolds moyen peut consommer plusieurs jours voire plusieurs semaines de temps de calcul sur une station de travail de haut de gamme.

ESAIM: PROC., VOL. 3, 1998, 37-50 


\section{Applications numériques}

Tous les tests présentés sont calculés à partir des équations pénalisées (13) (14) et utilisent les conditions aux limites (10) sur les limites artificielles autres que la section d'entrée sur laquelle est imposé l'écoulement amont. Les simulations en dimension deux correspondent à un domaine de calcul identique ou similaire à celui représenté figure 1. Les équations sont discrétisées comme indiqué ci-dessus en différences finies sur maillages décalés pour un schéma de Gear du troisième ordre en temps. La méthode de résolution à chaque pas de temps est une méthode multigrille avec relaxation maille à maille comme lisseur [5]. Pour valider l'ensemble de l'approximation, on commence par calculer l'écoulement non confiné derrière un cylindre pour lequel on dispose d'expériences physiques et d'une nombreuse littérature. Le domaine de calcul est le rectangle $(0,4) \times(0,1)$ incluant un cylindre de diamètre $d=0.2$ centré au point $(1,0.5)$. L'écoulement amont est constant $U_{a m}=(1,0)$ et imposé sur $\Gamma_{D}$; assurant un débit de 1 . Le nombre de Reynolds réel est donc égal à $R_{y}=R e \times d$. Le tableau 1 donne une comparaison du nombre de Strouhal $S_{t}=f d / U_{a m}$ où $f$ est la fréquence pour différentes valeurs du nombre de Reynolds $R_{y}$. Les résultats obtenus sur une grille fine de $256 \times 64$ points sont en parfaite harmonie avec ceux estimés par les physiciens [35]. ils montrent ainsi la précision du processus global conduisant au calcul des solutions approchées au moins pour des nombres de Reynolds modestes.

\begin{tabular}{|c|c|c|c|}
\hline$R e$ & $R_{y}$ & $S_{t}$ calculé & $S_{t}$ estimé dans $[35]$ \\
\hline 300 & 60 & 0.130 & 0.136 \\
\hline 500 & 100 & 0.164 & 0.164 \\
\hline 800 & 160 & 0.188 & 0.186 \\
\hline
\end{tabular}

\section{Tab. 1 - Comparaison du nombre de Strouhal}

Les tests qui suivent correspondent à l'écoulement confiné derrière un cylindre dans un canal. Le domaine de calcul est celui de la figure 1 où $D=(0, L) \times(0,1)$ avec $L=3$ ou $L=4$ et le cylindre de diamètre $d=0.4$ est centré au point $(1,0.5)$.



Fig. 3 - Solution à $R e=100$

Cette fois-ci l'écoulement amont est un écoulement de Poiseuille de débit 1 donné par $U_{a m}=\left(6 x_{2}\left(1-x_{2}\right), 0\right)$. Les tests sont effectués sur un maillage de $256 \times 64$ cellules et présentés en fonction du nombre de Reynolds adimensionné $R e$. Le nombre de Reynolds réel est donc déduit directement par $R_{y}=R e \times d$ et donc $R e=100$ correspond à $R_{y}=40$. Pour cette valeur, la solution est stationnaire et symétrique par rapport à l'axe $x_{2}=0.5$. On observe une zone de recirculation derrière le cylindre qui augmente en fonction de $R e$, bien visible sur les isolignes de la fonction de courant (figure 3). Ensuite, quand Re croit, la solution stationnaire devient instable au profit d'une solution périodique qui reste stable 
pour une large plage de nombres de Reynolds. Au début, la zone de recirculation oscille entre le haut et le bas du cylindre. Ce qui permet de calculer précisément le nombre de Reynolds critique car il correspond à la perte de symétrie de la solution. Ceci est bien entendu lié à la géométrie très particulière.
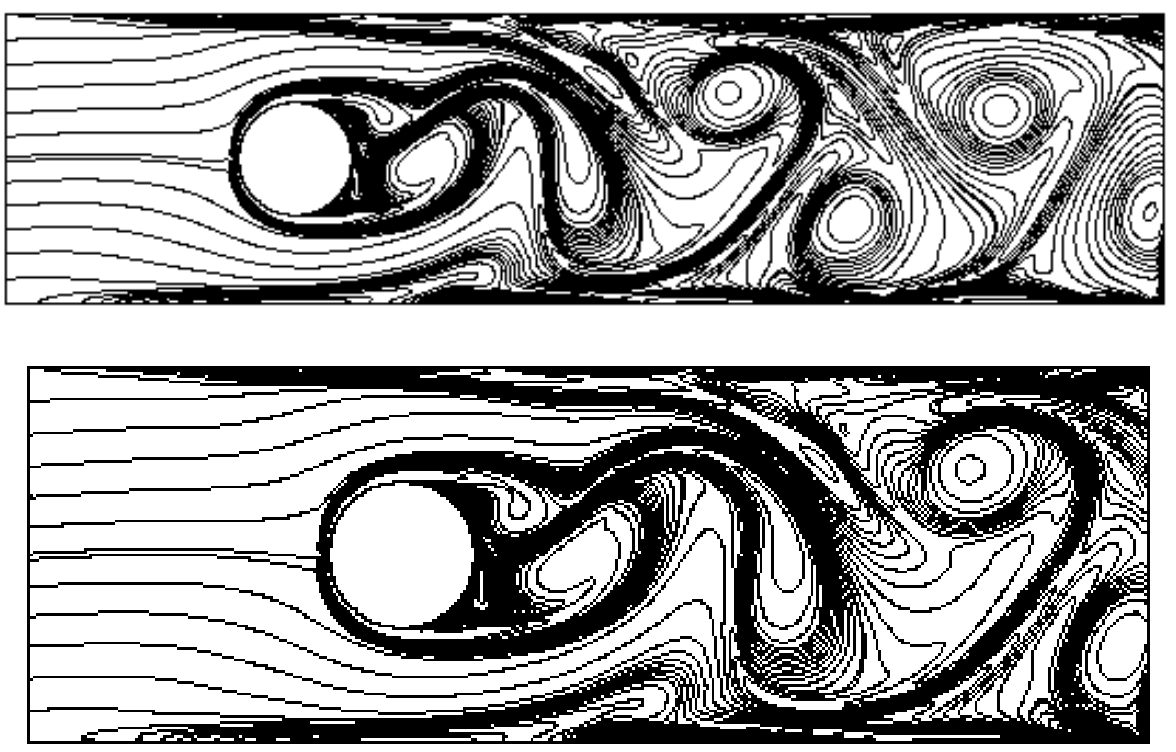

Fig. 4 - Comparaison des solutions obtenues sur le domaine $\Omega$ avec $L=4$ et $L=3$

Pour calculer des écoulements plus complexes, on utilise une grille fine de $512 \times 128$ mailles (la grille grossière comporte $16 \times 4$ mailles) et un pas de temps égal à $5.10^{-4}$. Pour $R e=1000$ par exemple l'écoulement reste périodique et on peut observer une allée de tourbillons qui sont convectés vers l'aval. C'est sur cette solution que nous mettons en évidence la pertinence des conditions aux limites (10). En effet la solution calculée sur un domaine plus court est quasi identique à la restriction de la solution calculée sur le grand domaine comme on peut le voir sur les lignes isotourbillons (figure 4). Il est à noter que les deux solutions sont calculées avec exactement les mêmes paramètres et que la simulation est faite exactement sur le même intervalle de temps. La seule différence est la longueur du domaine $L=4$ ou $L=3$. On voit en particulier qu'aucune réflexion ni aucun retard n'est induit par la coupure artificielle du domaine. Cela montre que les tourbillons, aussi gros soient-ils, sont convectés par l'écoulement à la bonne vitesse.

Quand on fait croitre le nombre de Reynolds, on observe une solution périodique jusqu'à $R e=3700$ mais dont la nature évolue. En effet, une analyse de Fourier montre qu'approximativement de $R e=200$ à $R e=3700$, l'écoulement est périodique avec la même fréquence fondamentale $f_{m} \simeq 1$ qui dépend des différents paramètres (figure 5). Mais de $R e=2200$ à $R e=3600$, on voit apparaitre deux sous harmoniques correspondant à $f \simeq \frac{f_{m}}{3}$ et $\frac{2 f_{m}}{3}$. Enfin pour $R e=3700$ il apparait sept sous harmoniques correspondant à $f \simeq \frac{f_{m}}{8}$ et ses multiples (figure 5).

L'apparition de ces sous harmoniques pourrait correspondre à la fusion de plusieurs tourbillons pour constituer une allée tourbillonnaire de von Karman. Il est clair que ce comportement qualitatif dépend fortement de la géométrie du problème. En effet, le fait de remplacer le cylindre à section circulaire par un cylindre à section carrée de côté $d=0.4$ change le nombre de sous harmoniques. Pour tous les tests qui précèdent, une analyse de Fourier s'est 
A
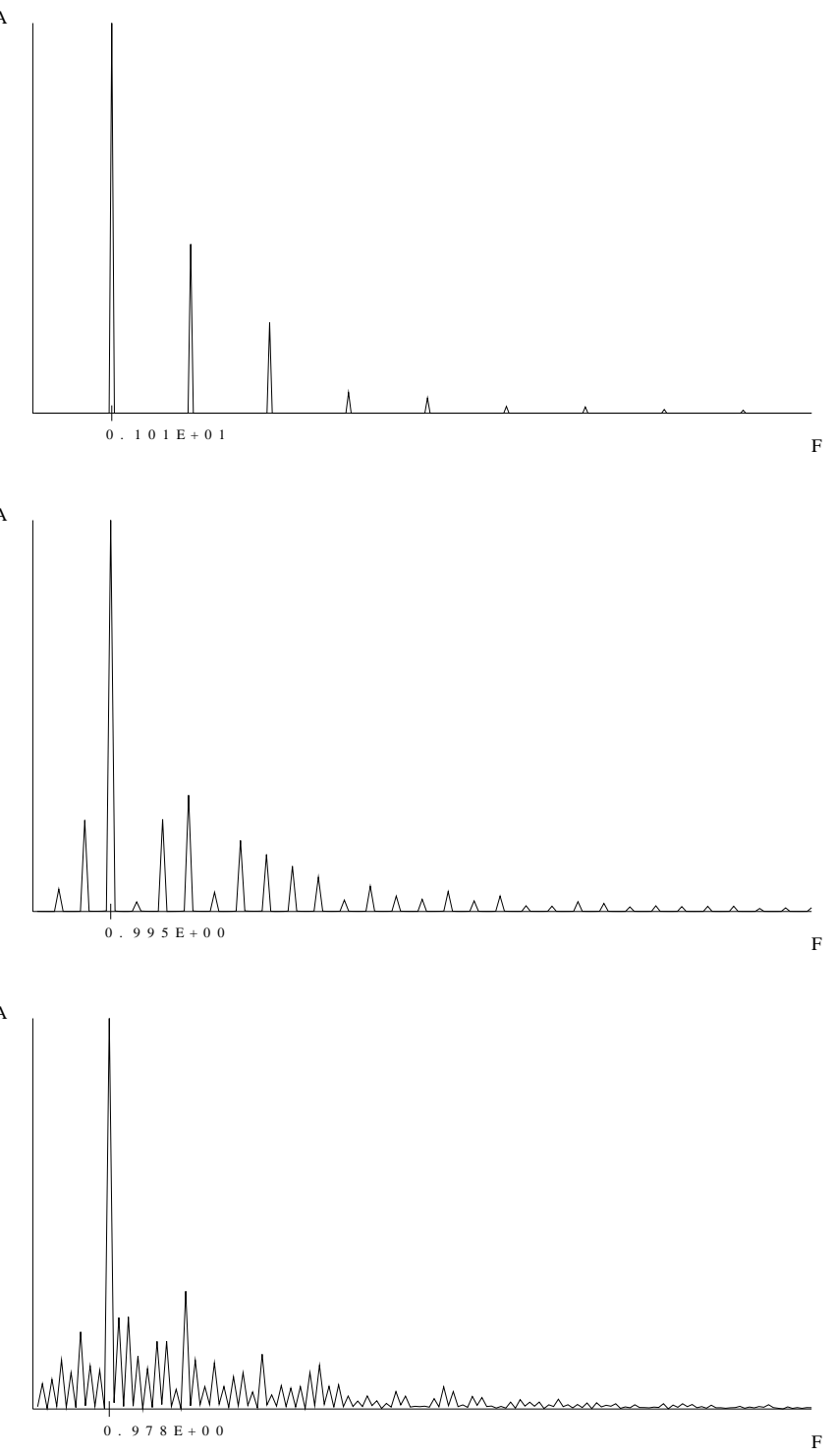
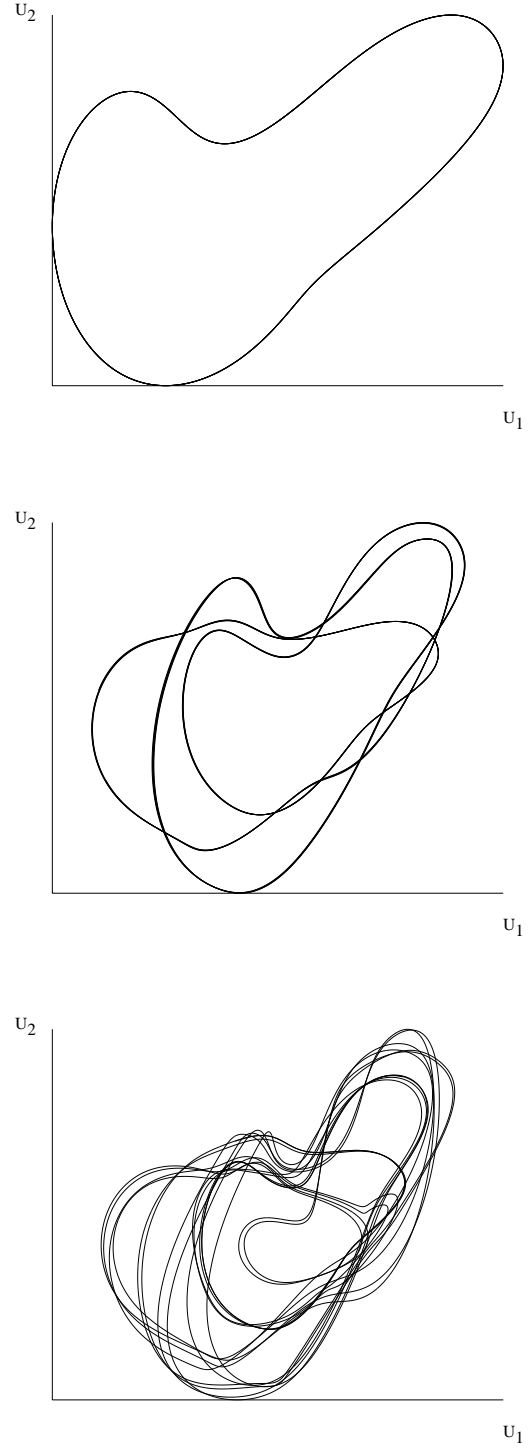

FIG. 5 - Spectre et portrait de phase pour $R e=1000$, 2500 et 3700 
révélée très efficace pour analyser les signaux temporels enregistrés en un point quelconque du domaine choisi en aval de l'obstacle. De plus les résultats obtenus en analysant la vitesse en divers points sont identiques. Une vue du portrait de phase donne une information visuelle immédiate qui corrobore les résultats de l'analyse. En effet la courbe fermée triple à $R e=2500$ montre clairement la présence des deux sous harmoniques (figure 5). Par contre une analyse en ondelettes ne s'avère pas plus riche en informations et est moins précise sur la détermination des fréquences. On peut voir sur la figure 6 qu'elle détecte bien la même fréquence fondamentale et les mêmes sous harmoniques de la solution à $R e=3700$.

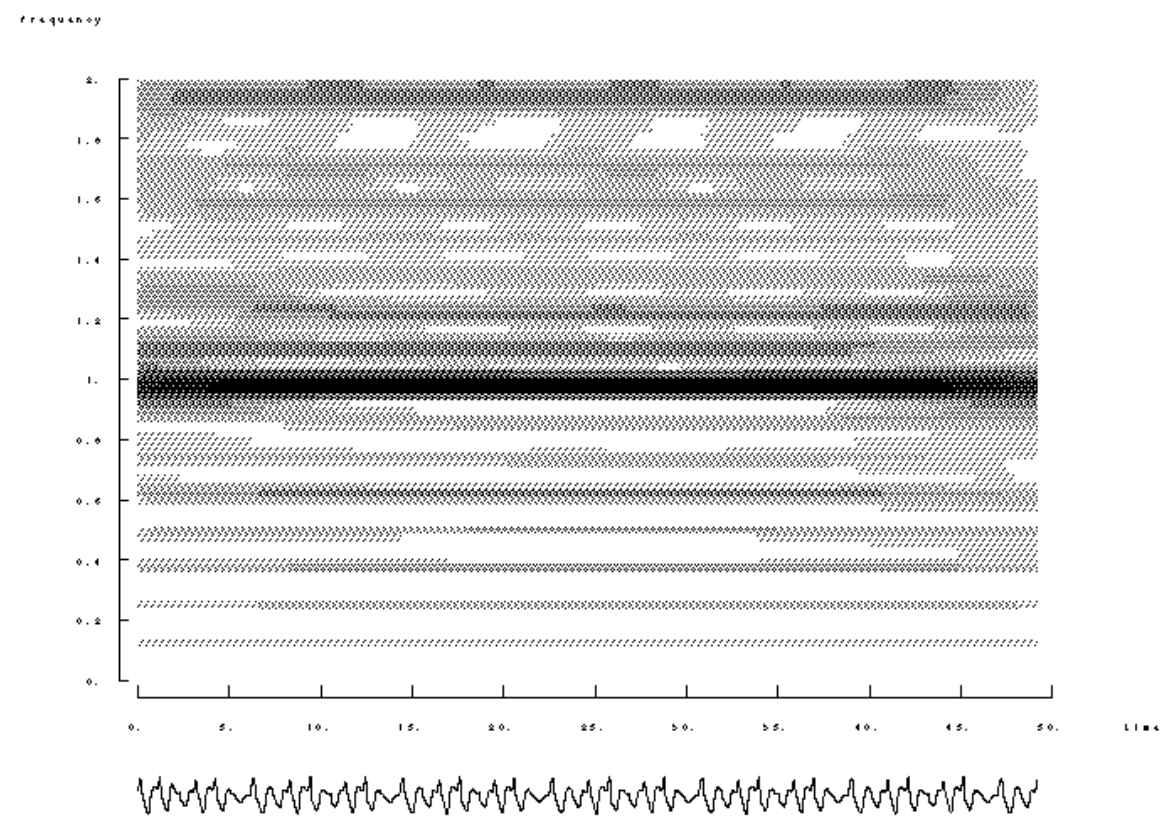

Fig. 6 - Analyse en ondelettes du signal à Re $=3700$

A $R e=3800$, une simulation sur un temps long $(T=200)$ montre que la solution alterne entre deux etats. Il est bien connu que les ondelettes sont a priori idéales dans ce cas de figure $[8,24,29]$ mais ici la transition entre les deux états est lente et correspond pratiquement à un morceau de spectre continu. Aussi l'analyse est très délicate et les résultats obtenus avec une transformation de Fourier à fenêtre ou une transformation en ondelettes sont comparables et un peu décevants dans la mesure où ils n'apportent pas d'information supplémentaire à ce que l'œil perçoit sur le signal temporel (figure 7).

Pour de plus grands nombres de Reynolds la solution devient chaotique. On voit clairement la présence de structures désordonnéees sur la figure 8 avec des zones de forte activité et des zones de repos révélées par les lignes isotourbillons. L'analyse du signal temporel n'apporte pas beaucoup d'éléments nouveaux. La fréquence fondamentale semble toujours présente avec quelques variations au cours du temps. Le spectre s'est épaissi, en particulier vers les basses fréquences et le plan de phase ressemble à un plat de nouilles. Une analyse plus fine en ondelettes ne donne aucune indication supplémentaire. Un algorithme de type matching pursuit [22] pourrait peut-être apporter quelques informations à condition de trouver le bon dictionnaire. Un autre aspect de l'analyse est de suivre les structures au cours du temps pour mettre en évidence la fusion ou l'éclatement de tourbillons. Il y a eu quelques travaux en ondelettes pour comprimer des écoulements turbulents en dimension deux [11]. Les 




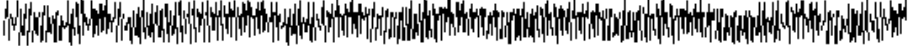

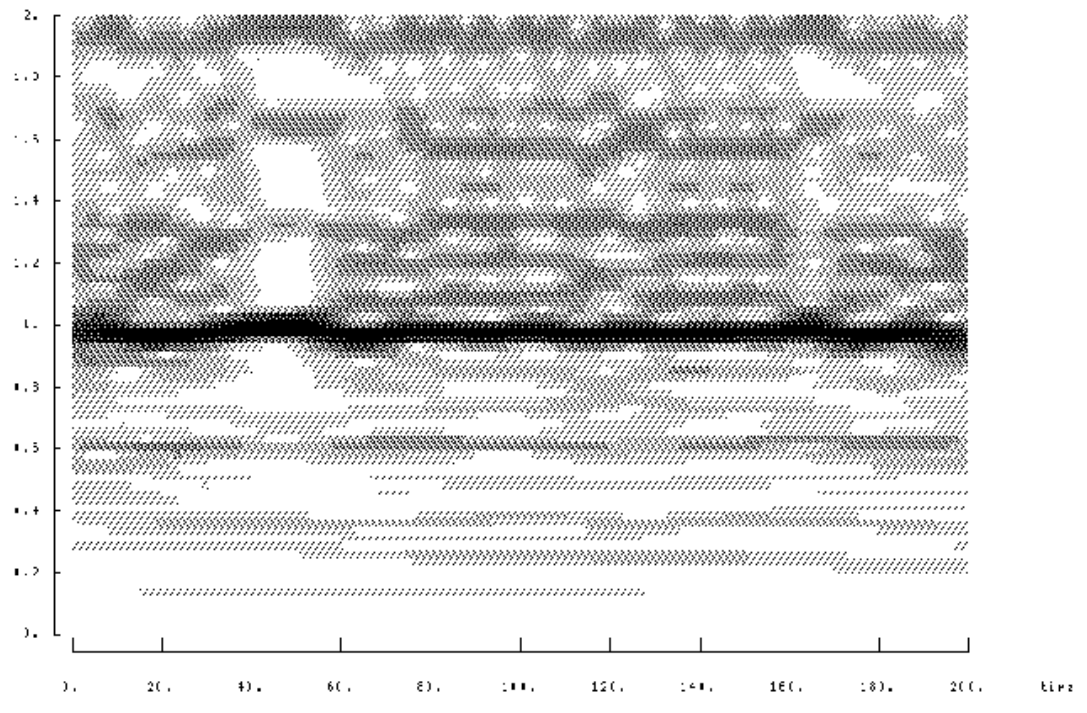

Fig. 7 - Analyse de Fourier à fenêtre et en ondelettes du signal à Re $=3800$

ESAIM: PROC., VOL. 3, 1998, 37-50 
résultats montrent qu'il est possible de capturer les structures essentielles, reste à voir si on peut raisonnablement les suivre au cours du temps et en tirer des informations pertinentes.

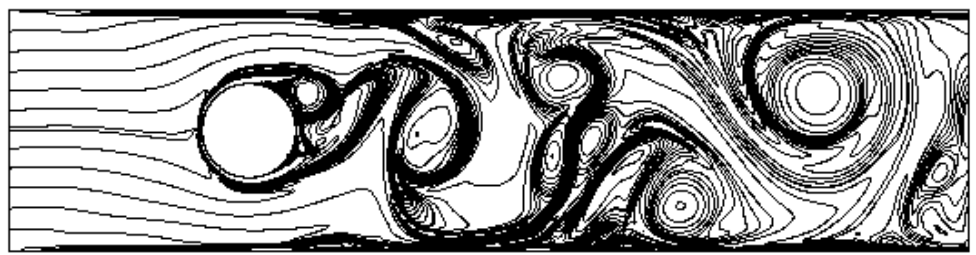

FIG. 8 - Solution à $R e=10000$

Le calcul d'écoulements transitoires ou turbulents en dimension deux est souvent remis en question car la turbulence est réputée par essence tridimensionnelle. Une rencontre récente avec Hamid Kellay nous a montré que de nombreux physiciens travaillent sur la turbulence 2D. Lui fait en particulier une expérience qui consiste à construire un écoulement bidimensionnel. Il s'agit d'un film de savon de cinq centimètres de largeur s'écoulant entre deux fils de pêche verticaux tendus. La régulation du débit permet de couvrir une plage importante de nombres de Reynolds. Il est alors possible de visualiser des écoulements turbulents derrière l'obstacle de son choix. L'épaisseur du film varie en fonction des structures turbulentes et est considérée comme un traceur passif au même titre qu'un panache de fumée. En faisant l'hypothèse que le rôle de la tension superficielle est négligeable, nous avons simulé cette expérience pour une rangée de cinq cylindres de diamètre $d=0.1$ à une distance $d_{i}=0.1$ les uns des autres dans le domaine $D=(0,3) \times(0,1)$. On a alors $R_{y}=R e \times d$ pour comparer avec l'expérience. Les premiers résultats numériques montrent que l'écoulement présente les mêmes caractéristiques qualitatives: une première zone où l'on voit clairement les lachers de tourbillons alternés de taille $d$, une deuxième zone de tourbillons de taille $2 d$ et une zone plus éloignée d'allure chaotique (figure 9). Par ailleurs une première analyse statistique montre que la zone inertielle est bien capturée pour des modes supérieurs à la fréquence fondamentale et obéit pratiquement à la bonne loi de puissance [20, 36]. Il est à noter que la zone inertielle ne peut être correctement capturée que si le maillage (ou la troncature pour une méthode spectrale) le permet. Au delà, on n'obtient qu'une zone dissipative.

\section{Conclusion}

Il est aujourd'hui possible, surtout en dimension deux, de calculer des solutions transitoires à partir d'une simulation directe des équations de Navier-Stokes. Ces solutions donnent une bonne représentation qualitative de la transition vers le chaos mais leur sens quantitatif est beaucoup plus discutable. En utilisant au mieux les progrès de l'approximation et les capacités des ordinateurs, on peut augmenter le nombre de points nécessaires à la capture des principaux phénomènes et espérer calculer de bonnes solutions. Cependant, il est difficile avec les moyens d'analyse dont on dispose de bien les étudier.

\section{Remerciements}

Nous remercions vivement Roger Gay et Alain Yger avec qui nous avons eu de nombreuses discussions instructives sur l'analyse temps-fréquence, ainsi que Bruno Torrésani et JeanFrançois Delage pour les analyses de signaux en ondelettes. Une collaboration s'amorce avec Hamid Kellay qui réalise ces belles expériences sur les films de savon. Nous pensons qu'elle sera riche en enseignements. 


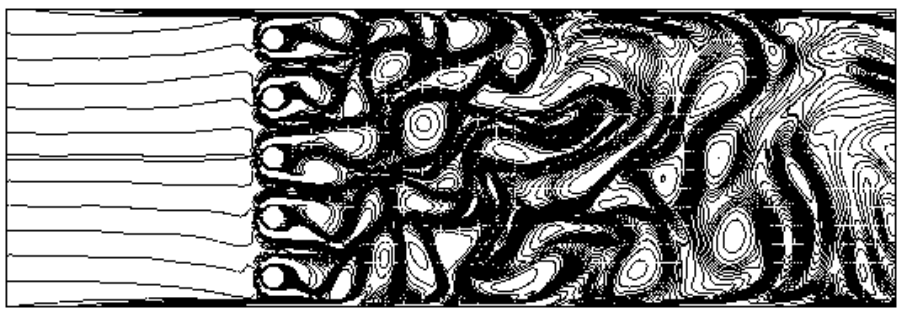

FIG. 9 - Ecoulement derrière cinq cylindres à $R_{y}=1000$

\section{Références}

[1] Ph. Angot, Ch.-H. Bruneau \& P. Fabrie, A penalization method to take into account obstacles in incompressible viscous flows, (soumis).

[2] A. Brandt, Multigrid techniques: Guide with applications to fluid dynamics, GMDStudien 85, (1984).

[3] C.-H. Bruneau \& P. Fabrie, New efficient boundary conditions for incompressible Navier-Stokes equations: A well-posedness result, $M^{2} A N \mathbf{3 0},(1996)$.

[4] C.-H. Bruneau \& P. Fabrie, Effective downstream boundary conditions for incompressible Navier-Stokes equations, Int. J. Num. Meth. Fluids 19, (1994).

[5] C.-H. Bruneau \& C. Jouron, An efficient scheme for solving steady incompressible Navier-Stokes equations, J. Comp. Phys. 89, nº 2, (1990).

[6] J.-P. Caltagirone, Sur l'interaction fluide-milieu poreux: Application au calcul des efforts exercés sur un obstacle par un fluide visqueux, $C R A S \mathbf{3 1 8}$, série 2, (1994).

[7] C. Canuto, M.Y. Hussaini, A. Quarteroni \& T.A. Zang, Spectral methods in fluid dynamics, Springer-Verlag, 1988.

[8] C. Chui, Wavelets: A tutorial in theory and applications, Academic Press, 1992.

[9] G. Danabasoglu, S. Biringen \& C.L. Streett, Application of the spectral multidomain method to the Navier-Stokes equations, J. Comp. Phys. 113, no2, (1994).

[10] S.M. Deshpande, S.S. Desai \& R. Narasimha (Eds), Numerical methods in fluid dynamics, Proceedings of the $14^{\text {th }}$ Int. Conf., Lecture notes in physics 453, 1995.

[11] M. Farge, E. Goiraud, Y. Meyer, F. Pascal \& M.V. Wickerhauser, Improved predictability of two-dimensional turbulent flows using wavelet packet compression, Fluid Dyn. Res. 10, 1992.

[12] C.A. Fletcher, Computational techniques for fluid dynamics, Vol.1 \& 2, SpringerVerlag, 1991.

[13] V. Girault \& P.-A. Raviart, Finite element methods for Navier-Stokes equations: Theory and algorithms, Springer-Verlag, 1986. 
[14] D. Goldstein, R. Handler \& L. Sirovich, Modeling a no-slip flow boundary with an external force field, J. Comp. Phys. 105, $n^{\circ} 2$, (1993).

[15] P.M. Gresho, Incompressible fluid dynamics: Some fundamental formulation issues, Ann. Rev. Fluid Mech. 23, (1991).

[16] W. HackBush, Multigrid methods and applications, Springer-Verlag, 1985.

[17] M. Hafez (Ed), Computational fluid dynamics, Proceedings of the $6^{\text {th }}$ Int. Symp., 1995.

[18] J.G. Heywood, K. Masuda, R. Rautwann \& S.A. Solarnikov (Eds), The Navier-Stokes equations - Theory and numerical methods, Proceedings of the $2^{\text {nd }}$ Conf., Lecture notes in math. 1530, 1992.

[19] Ch. Hinsch, Numerical Computation of internal and external flows, Vol. 1 \& 2, 1990.

[20] H. Kellay, X.L. Wu \& W.I. Goldburg, Experiments with turbulent soap films, Phys. Rev. Letters 74, no20, (1995).

[21] P. Le Quere \& T. Alziary de Roquefort, Computation of natural convection in two-dimensional cavities with Chebyshev polynomials, J. Comp. Phys. 57, (1985).

[22] S. Mallat \& Z. Zhang, Matching pursuits with time-frequency dictionaries, Report 619, Courant Institute, (1992).

[23] M. Marion \& R. Temam, Navier-Stokes equations: Theory and approximation, Handbook of Numerical Analysis, (à paraitre).

[24] Y. Meyer \& S. Roques (Eds), Progress in wavelet analysis and applications, Proceedings of the Int. Conf., 1992.

[25] M. Napolitano \& F. Sabetta (Eds), Numerical methods in fluid dynamics, Proceedings of the $13^{\text {th }}$ Int. Conf., Lecture notes in physics 414, 1993.

[26] S.V. Patankar, Calculation procedure for viscous incompressible flows in complex geometries, J. Numer. Heat Transfer 14, $n^{\circ} 3$, (1988).

[27] C.S. PEskin, Numerical analysis of blood flow in the heart, J. Comp. Phys. 25, (1977).

[28] R. Peyret \& T.D. Taylor, Computational methods for fluid flow, Springer-Verlag, 1983.

[29] M.B. Ruskai, G. Beylkin, R. Coifman, I. Daubechies, S. Mallat, Y. Meyer \& L. Raphael (Eds), Wavelets and their applications, Jones and Barlett Publishers, 1992.

[30] E.M. Saiki \& S. Biringen, Numerical simulation of a cylinder in uniform flow: Application of a virtual boundary method, J. Comp. Phys. 123, (1996).

[31] R.L. Sani \& P.M. Gresho, Résumé and remarks on the open boundary condition minisymposium, Int. J. Num. Meth. in Fluids, 18, (1994).

[32] C. Taylor, J.H. Chin \& G.M. Homsy (Eds), Numerical methods in laminar and turbulent flow, Vol. 7, Part $1 \&$ 2, Proceedings of the $7^{\text {th }}$ Int. Conf. 1991.

[33] F. Thomasset, Implementation of finite elements methods for Navier-Stokes equations, Springer-Verlag (1981).

[34] R. Temam, Navier-Stokes equations, North-Holland (1984).

[35] C.H. Williamson, Oblique and parallel modes of vortex shedding in the wake of a circular cylinder at low Reynolds numbers, J. Fluid Mech. 206 (1989).

[36] X.L. Wu, B. Martin, H. Kellay \& W.I. Goldburg, Hydrodynamic convection in a two-dimensional Couette cell, Phys. Rev. Letters $75 n^{\circ} 2$ (1995).

[37] N.G. Wright \& P.H. GaskelL, An efficient multigrid approach to solving highly recirculating flows, Comput. Fluids 24, $n^{\circ} 1$ (1995). 